\title{
Geographic Access and Maternal Health Services Utilization in Sélingué Health District, Mali
}

\author{
Moctar Tounkara ${ }^{1}$ (1) Oumar Sangho ${ }^{1,2} \cdot$ Madeleine Beebe $^{3} \cdot$ Lillian Joyce Whiting-Collins $^{3} \cdot$ Rebecca R. Goins $^{3}$. \\ Hannah C. Marker ${ }^{3} \cdot$ Peter J. Winch ${ }^{4} \cdot$ Seydou Doumbia ${ }^{1,5}$
}

Accepted: 10 December 2021 / Published online: 22 January 2022

(c) The Author(s), under exclusive licence to Springer Science+Business Media, LLC, part of Springer Nature 2021

\begin{abstract}
Introduction Maternal mortality is one of the main causes of death for women of childbearing age in Mali, and improving this outcome is slow, even in regions with relatively good geographic access to care. Disparities in maternal health services utilization can constitute a major obstacle in the reduction of maternal mortality in Mali and indicates a lack of equity in the Malian health system. Literature on maternal health inequity has explored structural and individual factors influencing outcomes but has not examined inequities in health facility distribution within districts with moderate geographic access. The purpose of this article is to examine disparities in education and geographic distance and how they affect utilization of maternal care within the Sélingué health district, a district with moderate geographic access to care, near Bamako, Mali.

Methods We conducted a cross sectional survey with cluster sampling in the Sélingué health district. Maternal health services characteristics and indicators were described. Association between dependent and independent variables was verified using Kendall's tau-b correlation, Chi square, logistic regression with odds ratio and 95\% confidence interval. Gini index and concentration curve were used to measure inequity.

Results Most respondents were 20 to 24 years old. Over $31 \%$ of our sample had some education, $65 \%$ completed at least four ANC visits, and 60.8\% delivered at a health facility. Despite this evidence of healthcare access in Sélingué, disparities within the health district contribute to inadequate utilization among approximately $40 \%$ of the women in our sample. The concentration index demonstrated the impact of inequity in geographic access,

comparing women residing near and far from the referral care facility.

Conclusion Maternal health services underutilization, within a district with moderate geographic access, indicates that deliberate attention should be paid to addressing geographic access even in such a district.
\end{abstract}

Keywords Inequity $\cdot$ Antenatal care $\cdot$ Caesarian section $\cdot$ Geographic access

\section{Abbreviations}

ANC Antenatal care

ABE Adult based education

Moctar Tounkara

moctartounkara5@gmail.com

1 Department of Public Health, Faculty of Medicine and Dentistry, University of Sciences, Techniques and Technologies of Bamako, Bamako, Mali

2 Agence Nationale de Télémédecine et d'Informatique Médicale, Bamako, Mali

3 MUSO NGO, San Francisco, USA

4 Department of International Health, Social and Behavioral Interventions Program, Johns Hopkins Bloomberg School of Public Health, Baltimore, MD, USA

5 University Clinical Research Center, Bamako, Mali
3-Es Woman's economic, education and empowerment status

LMICs Low- and middle-income countries

\section{Significance}

What is known? Geographic access to care is a major factor contributing to maternal mortality, given that many life-saving interventions like caesarian sections and blood transfusions are only available at referral health facilities. In moderate access geographic areas, large distances to health facilities for some residents contribute to elevated levels of direct obstetric mortality.

What does this study add? The importance of ensuring more evenly distributed health care facilities within 
moderate geographic access areas should not be neglected, simply because these districts already have better access relative to low geographic access regions.

\section{Introduction}

The African continent faces some of the highest rates of maternal mortality in the world, and maternal mortality is the leading cause of death in women of reproductive age in Mali. The largest cause of maternal death in Mali is hemorrhage; hemorrhage (Mallé et al., 1994) and obstructed labor require immediate attention. Women with severe anemia cannot afford to lose much blood before they go into shock, as they are starting off with lower hemoglobin levels (Brabin et al., 2001). After 2000, national-level estimates of the maternal mortality ratio began to decrease in Mali, dropping from 464 to 325 deaths per 100,000 live births between 2000 and 2018 (Cellule de Planification et de Statistique du Ministère de la Santé, 2018; Cellule de Planification et de Statistique Ministère de la Santé, 1996-2002, 2006). However, this sharp decline obscures much higher levels in more remote rural areas. A 2011 study showed that the maternal mortality ratio can be far higher in rural Mali than estimates based on nationally representative samples (Aa et al., 2011). Social-systemic factors contributing to the "neglected tragedy" of maternal mortality are inadequate access to care, under-utilization of modern healthcare services, and women's limited decision-making power, often linked to their education level (Simkhada et al., 2008).

Geographic access to care is a major factor contributing to maternal mortality, given that many life-saving interventions like caesarian sections and blood transfusions are only available at referral health facilities. In a secondary analysis of census data from moderate geographic access areas of Tanzania, large distances to hospitals contribute to high levels of direct obstetric mortality. Deaths due to direct causes of maternal mortality were strongly related to distance from a health facility, with mortality increasing from 111 per 100,000 live births among women who lived within $5 \mathrm{~km}$ to 422 deaths per 100,000 live births among those women who lived more than $35 \mathrm{~km}$ from a hospital (Hanson et al., 2015). Other causes of delay relating to low-income or geographically remote regions can be attributed to poor roads, lack of available vehicles, and transportation costs (Babinard \& Roberts, 2006). Even with coordinated transportation, long distances from remote regions to a health facility are themselves another delay, as the distance to travel to a facility may take multiple hours or even days. Unlike antenatal care (ANC) visits, emergency care during labor and delivery cannot always be planned, and this delay can make childbirth in a facility highly unlikely. This geographic barrier is represented by the second delay in the three delays model: (1) delays on decision making, (2) delays in reaching health facilities, and (3) delays in receiving appropriate care (Barnes-Josiah et al., 1998).

The underutilization of ANC is another key factor impacting maternal mortality. Women in Mali typically do not initiate ANC visits early enough in their pregnancy. Furthermore, in maternal emergencies, they may not seek care from a health facility at all. According to a systematic review by Peters et al., access barriers can be identified in four dimensions, encompassing shortages in supply and demand: geographic accessibility, availability, affordability, and acceptability (Peters et al., 2008). For maternal mortality, factors associated with the use of ANC and delivery services are education, cost, quality of care, and access to services (Simkhada et al., 2008). These factors align with Peters et al.'s four dimensions of barriers to health services. Within this framework, this paper focuses on variations and equity in geographic access, in a district with moderate geographic access (compact district, close to the capital city of Bamako), compared to most other districts located farther from the capital.

Women's decision-making power is the third key factor that will be discussed. Women's autonomy increases directly as a function of their years of completed education and literacy, the cornerstone of sustainable development (Oyitso, 2012). More educated women delay marriage and childbearing, are more likely to use modern contraceptives, and thus are at less risk for pregnancy and maternal mortality (Larsson \& Stanfors, 2014). In a large-scale randomized study on adults who completed an adult based education (ABE), there was a significant drop in infant and maternal mortality among women of the same wealth group compared to those women who had never completed ABE (Lauglo, 2001). There is substantial evidence indicating that education and female literacy allow women to make better health decisions for themselves and their children, including accessing healthcare services such as vaccinations, contraception, childbirth, and postpartum care (Lauglo, 2001). Women's autonomy most directly affects the first of the 3-delays to seeking care: delays on decision making to seek care (Barnes-Josiah et al., 1998).

Throughout existing systematic analyses and reviews, the unequal distribution of healthcare facilities throughout low geographic access areas of high geographic access districts is rarely discussed. High geographic access areas and moderate geographic access areas have lower maternal mortality compared to areas with lowest geographic access (Aa et al., 2011). Despite this need, maternal health programs cannot forego efforts to improve access in assumed moderate geographic access areas, since the geographic distribution of those facilities in these regions, and women's access to them, is still inequitable. Simply constructing more health facilities within a district may not be sufficient (Noor et al., 
2006). Geographic access to health facilities is often overestimated due to the assumption that people always elect to go to their closest healthcare facility and that they travel in a straight line to that facility (Noor et al., 2006). A study in Kenya simulated the ease or difficulty of physical access to government health facilities through a GIS algorithm. Even within urban, high geographic access regions of Kenya, it was found that about four million people did not live as close to healthcare facilities as previously assumed (Noor et al., 2006). Barriers such as difficult terrain, and variation in services offered and quality of care from one facility compared to another illustrate that access varies more than just proximity to a facility on a map.

Gaps in education and distance from the facility are well documented as factors affecting ANC utilization and birth in healthcare facilities (Say \& Raine, 2007; Simkhada et al., 2008). These factors are well-known, yet it is unclear how geographic access varies within districts, and how that affects maternal health equity and healthcare utilization and access. Sections of Mali are categorized as high, moderate, and low geographic access, and within these high geographic access locations entire districts are designated as having high, moderate, or low access to maternal healthcare facilities. Areas are considered to have low geographic access largely by their population density (Ratcliffe et al., 2016). Districts having moderate geographic access have not been assessed on the disparities that exist within these area, and their corresponding variability of healthcare utilization.

This study examines maternal healthcare utilization within the Sélingué health district, a district with moderate geographic access. We examine two main social determinants of health services utilization: education and geographic distance from a healthcare facility. This research applied tools for analysis of equity in healthcare to examine disparities in education and geographic distance and how they affect access to maternal care throughout the health district of Sélingué.

\section{Methods}

\section{Study Site}

The study was conducted in the health district of Sélingué, located $145 \mathrm{~km}$ southwest of Bamako. A health district is a geographic zone defined by the Malian government to provide public and private integrated healthcare as well as supported services (laboratories, logistics, etc.). The study district is made up of 60 villages with a total of 91,425 inhabitants, and it is divided into seven sub-districts. Each sub-district has a community health center where women receive their antenatal and maternal care. The one referral care facility, the district hospital, is staffed by one or more physicians, while the sub-district health centers are run by a center technical director, usually a nurse, with the exceptions of Siékorolé and Diarani, where the health center is run by a physician.

\section{Sample}

We conducted a cross-sectional survey with cluster sampling proportional to village population size at two levels for a total of 30 clusters. The first stage was the randomized selection of the seven sub-districts, then the random selection of the villages within the chosen sub-districts.

At the last stage, we conducted random sampling to select the families in the village that included at least one eligible woman. Women were eligible to participate in the study if:

- They gave voluntary, free, and informed consent to participate in the study.

- They had given birth in the 12 months prior to the day of the survey, regardless of the outcome of the pregnancy, in accordance with the Demographic and Health Survey (Cellule de Planification et de Statistique du Ministère de la Santé, 2018).

- They had been residing in the randomly selected area for at least 6 months prior to the day of the survey.

The number of clusters per village depended on the population size. Thus, villages with a relatively high population had more clusters than villages with a lower population. In total, we selected 960 households, or 33 households per cluster, with at least one eligible woman in each household.

\section{Data Collection}

The protocol was approved by the Institutional Review Board of the Johns Hopkins Bloomberg School of Public Health of Baltimore, and the Faculty of Medicine and Pharmacy at the University of Sciences, Techniques and Technologies of Bamako. Physical risks to study participants were negligible. Overall, this was a minimal risk study that did not involve any administration of medications or other substances, medical or surgical procedures, and no biological samples were collected. All investigators were trained in data collection techniques, including a module on the protection of human subjects, informed consent, and maintaining confidentiality. A written consent form was signed and dated by interviewees and the investigators.

We conducted face-to-face interviews using a questionnaire. All investigators were trained in data collection techniques including a module on the protection of human subjects, informed consent and maintaining confidentiality. We collected data on the age of the women, the level of instruction, the parity, the distance between the village and 
the health facility, the number of ANC visits and the age of pregnancy at the first ANC visit.

Data was collected with Samsung Galaxy tablets, sent to an online server (Magpi), and extracted using Microsoft Excel. We established a standardized description of the characteristics and indicators of maternal health service utilization. After quality control and correction, excel spreadsheets were merged into a single SPSS database.

According to the Mali 2018 National Health Information System, $57 \%$ of the population is within $5 \mathrm{~km}$ of a health facility (Ministère de la Santé et de l'Hygiène Publique, 2018), however, we classified geographic access areas in the following three categories:

- High geographic access areas: At least 75\% of the population is within $5 \mathrm{~km}$ of a health facility

- Moderate geographic access areas: from 50 to $74 \%$ of the population is within $5 \mathrm{~km}$ of a health facility

- Low geographic access areas: less than $50 \%$ of the population is within $5 \mathrm{~km}$ of a health facility

\section{Data Analysis}

We performed an analysis between dependent and independent variables using simple and multiple logistic regression Chi-square tests to measure the determinants of service utilization. The gross and adjusted odd ratios were calculated with a $95 \%$ confidence interval and a p value of $0.05 \%$ for a significant difference. We verified the association between dependent variables and independent variables that have more than two modalities by using Kendall's tau-b correlation. Variables that had a significant association during bivariate analysis were put into the model.

To measure equity in geographic access, we calculated the Gini index and constructed a concentration curve. The concentration curve presents a visual graph of inequity in the use of health care and compares the level of inequity over distance. The Gini index is a quantitative measure of inequity in the use of healthcare, defined as "twice the area between the concentration curve and the equity line, to measure the degree of inequity systematically associated with distance" (Wagstaff et al., 1991). These two methods are standard measures to estimate inequity related to a variable on various health indicators (Babinard \& Roberts, 2006). We interpreted the inequity measured through the concentration curve and the concentration index as horizontal inequity, since all women were assumed to have the same maternal health needs, e.g. need for delivery in a referral health care facility, regardless of baseline characteristics (Zere et al., 2010). While the concentration curve is a useful tool for graphically representing inequity, it does not quantify the magnitude of the inequity.
Economic status was measured using the wealth index model taken from the Bangladesh Demographic and Health Survey (Rutstein \& Johnson, 2004). Geographic accessibility was estimated using the index of distance between a women's village of residence and local health facilities by using the concentration curve and the concentration index (distance index from place of residence to health facility). This index places women's villages of residence individually on a continuous scale of relative distance. Five quintiles of distance were used to categorize distance of the women to their health facility and to measure its influence on their maternal health indicators. Both principal components and factor analysis were carried out (Hossain, 2010). The value of the concentration index is between -1 and +1 . A value of 0 indicates that the use of health services is equitably distributed among socio-economic groups. The value of the distance index below zero indicates that women in remote places of residence use more health facilities than women in areas further from health facilities. A value above zero implies that women residing furthest from a facility use health services less than women who reside closer (Wagstaff et al., 1991).

\section{Results}

Table 1 provides an overview of the socio-demographic characteristics of women of childbearing age in Sélingué. In Table 1 , young women in the 20 to 24 -year age group were the most represented in the sample. Three-quarters of women reside in villages within $5 \mathrm{~km}$ of a first-level health facility, most women in this study $(60.7 \%)$ have no education at all, and $61.0 \%$ of women have at least three children.

In both Tables 2 and 3, data indicate that women living in villages between 6 to $15 \mathrm{~km}$ from a community health facility are 50\% less likely to achieve four or more ANC visits during pregnancy and $80 \%$ less likely to deliver in a health facility compared to women residing in villages located within $5 \mathrm{~km}$ of a community health facility $(\mathrm{p}<0.001)$. Women residing in villages more than $15 \mathrm{~km}$ from a community health facility are $40 \%$ less likely to achieve four or more ANC visits throughout pregnancy and are 90\% less likely to deliver in a community health facility, compared to those women residing in villages within $5 \mathrm{~km}$ of a first-level health facility $(\mathrm{p}<0.05)$.

Table 2 reveals that women who started their first ANC visit at 4 months or less of pregnancy are five times more likely to have four or more ANC visits during their entire pregnancy, compared to women who started the first ANC at 5 months or more of pregnancy $(\mathrm{p}<0.001)$.

Table 3 presents the odds ratio of health facility delivery with women's education levels and distance from a health facility. Women with up to 6 years of primary 
Table 1 Sociodemographic characteristics of women of childbearing age in Sélingué District, Mali

\begin{tabular}{|c|c|c|}
\hline Sociodemographic variables & $\mathrm{n}$ & $\%$ \\
\hline Age intervals (years) & 1021 & \\
\hline $15-19$ & 247 & 24.2 \\
\hline $20-24$ & 268 & 26.2 \\
\hline $25-29$ & 212 & 20.8 \\
\hline $30-34$ & 154 & 15.1 \\
\hline $35-39$ & 103 & 10.1 \\
\hline $40-49$ & 37 & 3.6 \\
\hline Level of education & 1021 & \\
\hline No formal instruction & 701 & 60.7 \\
\hline Primary schooling & 213 & 20.9 \\
\hline Second level of primary school & 88 & 8.6 \\
\hline At least secondary level & 19 & 1.9 \\
\hline Parity & 1021 & \\
\hline Primiparity & 199 & 19.5 \\
\hline Second parity & 197 & 19.3 \\
\hline Multiparity & 625 & 61.2 \\
\hline \multicolumn{3}{|l|}{ Geographic access } \\
\hline $\begin{array}{l}\text { Distance from village of residence to first- } \\
\text { level health facility }\end{array}$ & 1021 & \\
\hline $0-5 \mathrm{~km}$ & 749 & 73.4 \\
\hline $6-15 \mathrm{~km}$ & 169 & 16.6 \\
\hline$>15 \mathrm{~km}$ & 103 & 10.1 \\
\hline $\begin{array}{l}\text { Distance from nearest first-level health } \\
\text { facility to referral hospital }\end{array}$ & 1021 & \\
\hline $0-5 \mathrm{~km}$ & 219 & 21.4 \\
\hline $6-15 \mathrm{~km}$ & 210 & 20.6 \\
\hline$>15 \mathrm{~km}$ & 592 & 58.0 \\
\hline
\end{tabular}

education are twice as likely to give birth in a health facility, compared to women with no education $(p<0.01)$. Women with 6 to 9 years of education are 3.3 times more likely to give birth in a health facility compared to those women with no education $(\mathrm{p}<0.05)$.
Figure 1 shows that completion of four or more ANC visits during pregnancy is unequally distributed in favor of women from villages closest to a health facility. The farther the curve deviates from the perfect equity line, the greater the degree of inequity. In both Figs. 1 and 2, the concentration curve is below the equity line. Figure 2 shows that delivery in a health facility is also unequally distributed, in favor of women who reside in villages closest to a health facility.

\section{Discussion}

The sixth Demographic and Health Survey in Mali reports an increase in four or more ANC visits over the past two decades: from $43 \%$ of women in 2001 to $68 \%$ in 2018 . Furthermore, considerable progress has been made for the percentage of live birth deliveries in a health facility, increasing from 38 to $67 \%$ in the same time period (Cellule de Planification et de Statistique du Ministère de la Santé, 2018). Despite these improvements in healthcare utilization, maternal mortality remains a major cause of death among Malian women (Cellule de Planification et de Statistique du Ministère de la Santé, 2018).

The district of Sélingué is about $1.5 \mathrm{~h}$ away from the capitol of Bamako, and there is overall moderate geographic access to healthcare facilities. However, even within Sélingué, there is variation that has real impacts on usage of maternal healthcare and facilities.

Access to care and the underutilization of healthcare are key factors that impact maternal mortality (Simkhada et al., 2008). Yet in the health district of Sélingué, close to the national capital, with moderate geographic access to health services, there are clear disparities within the district. As noted in existing studies, the unequal distribution of healthcare facilities according to geographical accessibility in West African regions is rarely discussed.

(Abolhallaje et al. 2014; Awoyemi et al., 2011; Chen et al., 2014; Umar \& Bolanle, 2015). The internal disparities
Table 2 Women with at least four ANC visits with geographic accessibility and individual characteristics

\begin{tabular}{|c|c|c|c|c|c|}
\hline \multirow[t]{2}{*}{ Characteristics } & \multirow[t]{2}{*}{$\mathrm{N}$} & \multicolumn{2}{|c|}{$\begin{array}{l}\text { At least four ANC } \\
\text { visits }\end{array}$} & \multirow[t]{2}{*}{$\%$ Yes } & \multirow[t]{2}{*}{ OR (CI 95\%) } \\
\hline & & Yes (n) & No $(n)$ & & \\
\hline Gestational age at first ANC visit & 876 & 656 & 220 & & \\
\hline 5 months and more & 320 & 177 & 143 & 55.3 & 1 \\
\hline 4 months or less & 556 & 479 & 77 & 86.2 & $5.0 * * *(3.627-6.965)$ \\
\hline $\begin{array}{l}\text { Distance from village to first-level } \\
\text { health facility }\end{array}$ & 896 & 666 & 230 & & \\
\hline $0-5 \mathrm{~km}$ & 678 & 528 & 150 & 77.9 & 1 \\
\hline $6-15 \mathrm{~km}$ & 138 & 85 & 53 & 61.6 & $0.5 * * *(0.309 ; 0.672)$ \\
\hline$>15 \mathrm{~km}$ & 80 & 53 & 27 & 66.2 & $0.6^{*}(0.339 ; 0.917)$ \\
\hline
\end{tabular}

$* \mathrm{p}<0.05 ; * * \mathrm{p}<0.01 ; * * * \mathrm{p}<0.001$ 
Table 3 Health facility delivery comparing instruction level and distance to first-level health facility

\begin{tabular}{lrrrrl}
\hline Characteristics & $\mathrm{N}$ & \multicolumn{2}{l}{ Health facility delivery } & \% Yes & OR (CI 95\%) \\
\cline { 3 - 4 } & & Yes (n) & No (n) & & \\
\hline Instruction level & 1021 & & & & \\
$\quad$ No formal instruction & 701 & 405 & 296 & 86.4 & 1 \\
Primary school & 213 & 145 & 68 & 68.1 & $1.6^{* *}(1.113 ; 2.16)$ \\
Second-level of primary school & 88 & 84 & 4 & 68.1 & $1.2(0.74 ; 1.99)$ \\
At least secondary level & 19 & 11 & 8 & 57.9 & $0.6(0.25 ; 1.65)$ \\
Distance from village to first level of & 1021 & & & & \\
health facility & 749 & 535 & 214 & 71.4 & 1 \\
0-5 km & 169 & 62 & 107 & 36.7 & $0.2^{* * *}(0.16 ; 0.33)$ \\
6-15 km & 103 & 24 & 79 & 23.3 & $0.1 * * *(0.07 ; 0.19)$ \\
$>15 \mathrm{~km}$ & & & & & \\
\hline
\end{tabular}

$* \mathrm{p}<0.05 ; * * \mathrm{p}<0.01 ; * * * \mathrm{p}<0.001$

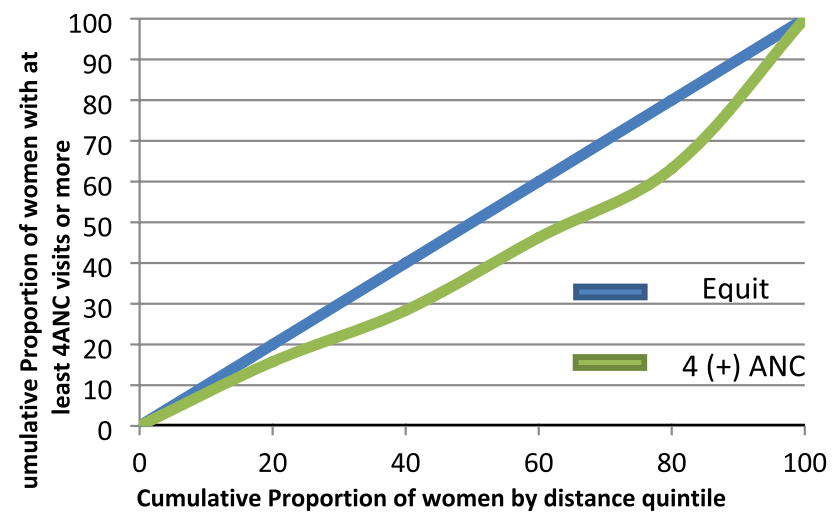

Fig. 1 Concentration curve of four or more ANC visits

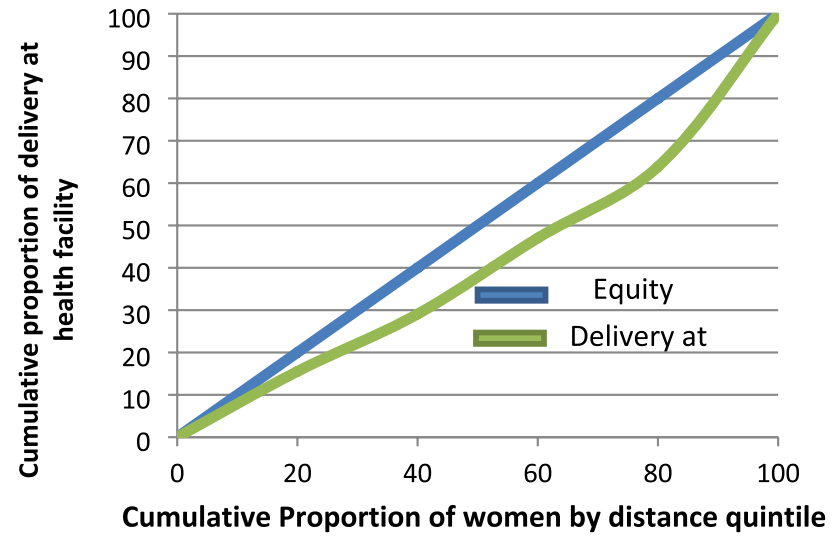

Fig. 2 Concentration curve of delivery at health facility

identified in this paper provide insight into why some women still experience poor maternal healthcare utilization and access, despite living in moderate geographic access regions of Mali. As depicted in Figs. 1, 2 and 3, the concentration curve demonstrates a lack of equity of access to delivery by healthcare facility and for women receiving four

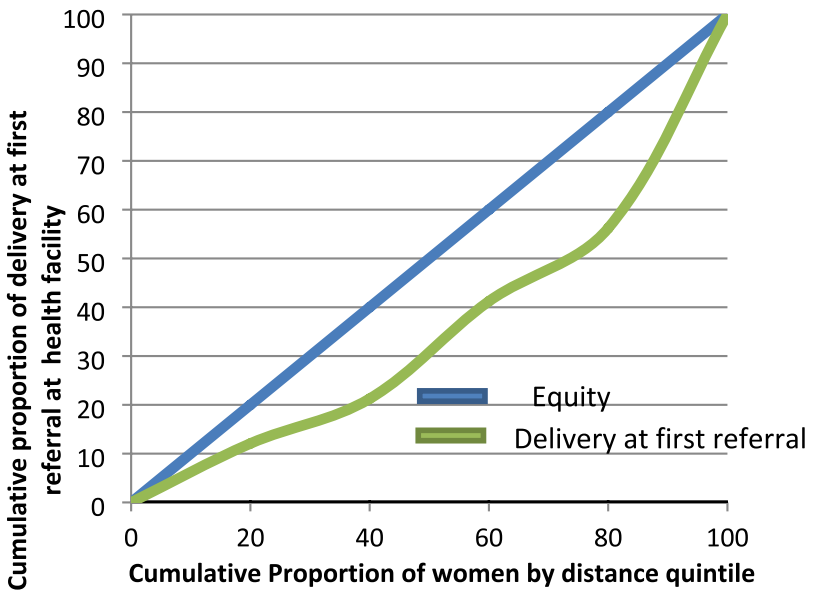

Fig. 3 Concentration curve of delivery at first referral of health facility

or more ANC visits. Gaps in education and distance from the facility are well documented as factors affecting ANC utilization and birth in healthcare facilities (Caliskan et al., 2015; Larsson \& Stanfors, 2014; Simkhada et al., 2008). However, the provided data highlights how geographic access varies within this moderate geographic access district, and how that affects maternal health equity and utilization access in Sélingué.

Women still face substantial barriers in the health district of Sélingué, despite this region having many health facilities. The distribution of these health facilities is not conducive to the equitable access of healthcare by all women in the district. A sizeable portion are still not delivering in a health facility or receiving ANC, and this inaccessibility is based on their distance from the facility, among other social factors.

Completion of a woman's first ANC visit in the earliest stages of her pregnancy (the first 4 months) is significantly associated with the completion of four or more ANC 
visits. Additionally, older pregnant women are more likely to complete four ANC visits. In addition to this timing, distance represents further disparity. The chance for a pregnant woman to have four or more prenatal visits decreases beyond a distance of $15 \mathrm{~km}$ between her home and a first-level health facility. As the distance between the health facility and a woman's village of residence decreased, her likelihood of attending four ANC visits increased.

The importance of evenly distributed health care facilities within moderate geographic access areas should not be neglected simply because they already have better access than low geographic access regions. The data tables demonstrate that if women live in villages between 6 and $15 \mathrm{~km}$ from a community health facility, they are 50\% less likely to achieve four or more ANC visits during pregnancy and $80 \%$ less likely to deliver in a health facility (Tables 2,3 ). Distributing health facilities more equally, or other efforts to bring care closer to women, can improve ANC services utilization in these more moderate geographic access areas. The findings reinforce that addressing utilization barriers is necessary in order to comply with the WHO recommendations for achievement of four or more ANC visits, facilitating childbirth in a health facility, and promoting equitable distribution of maternal health care and services (World Health Organization (WHO), 2016).

In addition to even facility distribution, attention should be given to gender equality and women's unequal access to education. A study completed by Ahmed et al., describes a concept similar to the 3-delays model but called the 3-Es (Ahmed et al., 2010). This study recognizes a woman's economic, education, and empowerment status as key indicators to maternal healthcare utilization (Ahmed et al., 2010). As supported in our data, women who are experiencing inequities in our education variable have lower healthcare service utilization (Table 3). Our data points to the larger socio-structural disadvantages that women face, and must be addressed country-wide, not only in Sélingué, to see longterm progress in women's maternal health. Efforts and interventions should be centered around gender equality by virtue of girl's education to improve women's overall maternal health as well as increase their utilization of health services.

Attention must continue to be placed in moderate geographic access areas such as the health district of Sélingué. Interventions should focus on addressing the determinants of the usage of equitable maternal healthcare, such as the 3-Es and distance to health facilities. Addressing these inequities will position Mali to be capable of achieving the third Sustainable Development Goal: to ensure healthy lives and promote wellbeing for all, at all ages.

Information and Communication Technologies (ICT) or tele-medicine can improve the equity in health care utilization in remote areas like Sélingué. (Bagayoko et al., 2010, 2011; Bediang et al., 2011). In the comparative study conducted in two rural health districts in Mali, the use of maternal health care increased due to the utilization of telemedicine (Bagayoko et al., 2011, 2014). In the context of the health district of Selingué, where distance constitutes a barrier to the use of maternal health services, telehealth can constitute a strategy for improving the use of services. However, this strategy will require the installation of infrastructure such as internet connection, computer equipment and a source of energy.

Our results align with previous studies that show higher educational attainment as significant in influencing birth in a health facility (Caliskan et al., 2015; Wong et al., 2017). Additionally, distance beyond $5 \mathrm{~km}$ of a health facility was significantly associated with a decrease in health facility utilization. There are some studies that contrast with our findings in their analysis of inequalities in the use of maternal care. In a range of low- and middle-income countries (LMICs), with particular attention to the low, moderate and high geographic access areas disparities, the association between place of residence and receipt of early ANC was not consistent (Say \& Raine, 2007). Some LMICs, like India, did not show a significant difference in ANC when comparing high geographic access areas and low geographic access areas with respect to their distance from health facility. However, it stands undisputed that antenatal care, childbirth, and postpartum care provided by a health facility are key factors in preventing maternal and neonatal mortality (Cellule de Planification et de Statistique du Ministère de la Santé, 2018).

In addition to distance as a barrier to the use of maternal health services, the Covid-19 pandemic has impeded the use of these services. (Oluoch-Aridi et al., 2020).

\section{Study Strengths and Weaknesses}

This research was conducted as a cross-sectional study with a retrospective survey. In this type of study design, there is a possibility for recall biases in our survey response subjects, which may affect our results of care-seeking behaviors. Additionally, a selection bias may also be at play since women who died during pregnancy, labor, or delivery before the survey was conducted were not included in the analysis.

\section{Suggestions for Further Research}

More research and in-depth assessments are needed to better address the interactions of these social determinants, and their impact on improving maternal healthcare utilization.

As noted in similar studies and systematic reviews, some countries of similar income levels do not see as stark disparities as Sélingué, Mali (Say \& Raine, 2007). If their inferences are true, possible points of additional research could be to articulate what allows for the mediation of 
these disparities. Particularly, what interventions, programs, or data are necessary to ensure a more equitable distribution of healthcare facilities and services in moderate geographic access areas. Other questions surround the impact of community-based transport systems and the construction of more referral care facilities that indirectly or directly impact the use of maternal health care.

During our proposal writing, we carried out an exchange visit to assess an obstetric emergency transportation system from villages to community health centers, operating in the Diema health district. Data analysis showed that the obstetric emergency transportation system from villages to community health centers can improve the use of delivery at health facilities.

\section{Conclusion}

This study analyzed the determinants of equity in the use of maternal health care in the Sélingue health district in Mali. Some studies assessed maternal health disparities associated with wealth index, maternal level of education, place of residence and administrative region (Say \& Raine, 2007), but rarely on disparities of maternal health services utilization associated with distance quintile in moderate geographic access regions.

In conclusion, our findings suggest that interventions and resources for gender equality and health facility access should be reallocated, with emphasis on their even distribution within the target area. Although there is critical need for greater health facility coverage in low geographic access areas, moderate and high geographic access areas also face disparities due to uneven access to facilities. The 3 -Es framework points to the value of supporting gender equality through social-structural changes improving maternal health indicators is not enough (Ahmed et al., 2010). In achieving the Sustainable Development Goals, it is necessary to:

- Improve the school enrollment rate of girls in low and moderate geographic access areas.

- Improve the coverage rate of maternal health care while reducing the distance of $15 \mathrm{~km}$ between villages and health facilities, especially in moderate geographic access areas.

With the support of many studies, including our own findings, the systemic benefits of increasing women's education and geographic distribution of facilities will result in improved maternal health and healthcare utilization, as well as economic mobility and thus large-scale social benefits (Ahmed et al., 2010).
Acknowledgements We would like to acknowledge the Johns Hopkins Bloomberg School of Public Health with his collaboration for this manuscript writing. We would also like to thank the Fogarty International Center for supporting Moctar Tounkara and Oumar Sangho under Grant D43TW008652. We thank Dr Lancina DOUMBIA and Aissata BA for the translation. We would like also to acknowledge the Sélingué district Health workers and the Sélingué district Health population.

Author Contributions MT prepared the first draft of the manuscript. All authors critically reviewed manuscript drafts.

Funding This study was funded through a NIH R21 Grant from the Fogarty International Center (Grant Number 1R21TW009885-01).

Data Availability The datasets used and/or analysed during the current study are available from the corresponding author on reasonable request.

\section{Declarations}

Conflict of interest The authors state that they have no competing interests.

Ethical Approval Ethical approval was received from the Faculty of Medicine and Odonto stomatology at the University of Sciences, Techniques and Technology of Bamako, Mali (REB number: 2015/112/ GE/FMPOS).

Consent for Publication Not Applicable.

\section{References}

Aa, I., Grove, M. A., Haugsjå, A. H., \& Hinderaker, S. G. (2011). High maternal mortality estimated by the sisterhood method in a rural area of Mali. BMC Pregnancy and Childbirth, 11(1), 1-6.

Abolhallaje, M., Mousavi, S. M., Anjomshoa, M., Nasiri, A. B., Seyedin, H., Sadeghifar, J., \& Nasiri, M. B. (2014). Assessing health inequalities in Iran: A focus on the distribution of health care facilities. Global Journal of Health Science, 6(4), 285.

Ahmed, S., Creanga, A. A., Gillespie, D. G., \& Tsui, A. O. (2010). Economic status, education and empowerment: Implications for maternal health service utilization in developing countries. PLoS ONE, 5(6), e11190.

Awoyemi, T. T., Obayelu, O. A., \& Opaluwa, H. I. (2011). Effect of distance on utilization of health care services in rural Kogi State, Nigeria. Journal of Human Ecology, 35(1), 1-9.

Babinard, J., \& Roberts, P. (2006). Maternal and child mortality development goals: What can the transport sector do? Retrieved June 30, 2020, from https://openknowledge.worldbank.org/handle/ 10986/17413.

Bagayokoa, C. O., Anneb, A., Fieschi, M., \& Geissbuhlera, A. (2011). Can ICTs contribute to the efficiency and provide equitable access to the health care system in Sub-Saharan Africa? The Mali experience. Yearbook of Medical Informatics, 20(01), 33-38.

Bagayoko, C. O., Niang, M., Traoré, S. T., Bediang, G. W., Naef, J. M., \& Geissbuhler, A. (2010). Deploying portable ultrasonography with remote assistance for isolated physicians in Africa: Lessons from a pilot study in Mali. Studies in Health Technology and Informatics, 160 (Pt 1), 554-558.

Bagayoko, C. O., Traoré, D., Thevoz, L., Diabaté, S., Pecoul, D., Niang, M., \& Geissbuhler, A. (2014). Medical and economic benefits of telehealth in low-and middle-income countries: Results 
of a study in four district hospitals in Mali. BMC Health Services Research, 14(1), 1-6.

Barnes-Josiah, D., Myntti, C., \& Augustin, A. (1998). The "three delays" as a framework for examining maternal mortality in Haiti. Social Science \& Medicine, 46(8), 981-993.

Brabin, B. J., Hakimi, M., \& Pelletier, D. (2001). An analysis of anemia and pregnancy-related maternal mortality. The Journal of Nutrition, 131(2), S604-S614.

Bediang, G. W., Bagayoko, C. O., Raetzo, M. A. E., \& Geissbuhler, A. (2011). Relevance and usability of a computerized patient simulator for continuous medical education of isolated care professionals in sub-saharan Africa. Studies in Health Technology and Informatics, 169, 666-670.

Çalışkan, Z., Kılıç, D., Öztürk, S., \& Atılgan, E. (2015). Equity in maternal health care service utilization: A systematic review for developing countries. International Journal of Public Health, 60(7), 815-825.

Cellule de Planification et de Statistique du Ministère de la Santé (CPS/ MS), Direction Nationale de la Statistique et de l'Informatique (DNSI) et ORC Macro. (2002). Enquête Démographique et de Santé au Mali 2001. CPS/MS, DNSI et ORC Macro.

Cellule de Planification et de Statistique du Ministère de la Santé (CPS/ MS), Direction Nationale de la Statistique et de l'Informatique du Ministère de l'Économie, de l'Industrie et du Commerce (DNSI/MEIC) et Macro International Inc. (2007). Enquête Démographique et de Santé du Mali 2006. CPS/DNSI et Macro International Inc.

Chen, Y., Yin, Z., \& Xie, Q. (2014). Suggestions to ameliorate the inequity in urban/rural allocation of healthcare resources in China. International Journal for Equity in Health, 13(1), 1-6.

Hanson, C., Cox, J., Mbaruku, G., Manzi, F., Gabrysch, S., Schellenberg, D., \& Schellenberg, J. (2015). Maternal mortality and distance to facility-based obstetric care in rural southern Tanzania: A secondary analysis of cross-sectional census data in 226000 households. The Lancet Global Health, 3(7), e387-e395.

Hossain, M. I. (2010). Inequality in the utilization of maternal care and the impact of a macroeconomic policy: Evidence from Bangladesh. The University of York.

Institut National de la Statistique (INSTAT), Cellule de Planification et de Statistique Secteur Santé-Développement Social et Promotion de la Famille (CPS/SS-DS-PF) et ICF. (2019). Enquête Démographique et de Santé au Mali 2018. INSTAT, CPS/SS-DS$P F$ et ICF.

Larsson, C., \& Stanfors, M. (2014). Women's education, empowerment, and contraceptive use in sub-Saharan Africa: Findings from recent demographic and health surveys. African Population Studies, 28(2), 1022-1034.

Lauglo, J. (2001). Engaging with adults: The Case for increased support to adult basic education in Sub-Saharan Africa. Retrieved June 30, 2020, from. http://www.worldbank.org/afr/hd/wps/ engage_adults.pdf.

Mallé, D., Ross, D. A., Campbell, O. M. R., \& Huttly, S. R. A. (1994). Institutional maternal mortality in Mali. International Journal of Gynecology \& Obstetrics, 46(1), 19-26.

Ministère de la Santé et de l'Hygiène Publique, Mali. (2018). Annuaire Statistique du Système National d'Information Sanitaire. Bamako. Document Number.
Noor, A. M., Amin, A. A., Gething, P. W., Atkinson, P. M., Hay, S. I., \& Snow, R. W. (2006). Modelling distances travelled to government health services in Kenya. Tropical Medicine \& International Health, 11(2), 188-196.

Oluoch-Aridi, J., Chelagat, T., Nyikuri, M. M., Onyango, J., Guzman, D., Makanga, C., \& Dowd, R. (2020). COVID-19 effect on access to maternal health services in Kenya. Frontiers in Global Women's Health, $1,19$.

Oyitso, M. (2012). Enhancing women's development through literacy education in Nigeria. Review of European Studies, 4, 66.

Peters, D. H., Garg, A., Bloom, G., Walker, D. G., Brieger, W. R., \& Rahman, M. H. (2008). Poverty and access to health care in developing countries. Annal of the New York Academy of Science, 1136(1), 161-171.

Ratcliffe, M., Burd, C., Holder, K., \& Fields, A. (2016). Defining rural at the US Census Bureau (ACSGEO-1). American Community Survey and Geography Brief, 1(8). Retrieved June 30, 2020, from https://www.researchgate.net/publication/311533270.

Rutstein, S. O., \& Johnson, K. (2004). The DHS wealth index. DHS comparative reports no. 6. Calverton: ORC Macro.. Retrieved January 2, 2018, from https://dhsprogram.com/pubs/pdf/cr6/cr6.pdf.

Say, L., \& Raine, R. (2007). A systematic review of inequalities in the use of maternal health care in developing countries: Examining the scale of the problem and the importance of context. Bulletin of the World Health Organization, 85, 812-819.

Simkhada, B., Teijlingen, E. R. V., Porter, M., \& Simkhada, P. (2008). Factors affecting the utilization of antenatal care in developing countries: Systematic review of the literature. Journal of Advanced Nursing, 61(3), 244-260.

Umar, J. U., \& Bolanle, W. (2015). Locational distribution of health care facilities in the rural area of Ondo State. Journal of Education, Society and Behavioural Science, 1-8.

Wagstaff, A., van Doorslaer, E., \& Paci, P. (1991). On the measurement of horizontal inequity in the delivery of health care. Journal of Health Economics, 10(2), 169-205.

Wong, K. L., Benova, L., \& Campbell, O. M. (2017). A look back on how far to walk: Systematic review and meta-analysis of physical access to skilled care for childbirth in sub-Saharan Africa. PLoS ONE, 12(9), e0184432.

World Health Organization. (2016). WHO recommendations on antenatal care for a positive pregnancy experience. World Health Organization. Retrieved September 28, 2016, from http://apps. who.int/iris/bitstream/10665/44369/1/9789241564014_eng.pdf.

Zere, E., Tumusiime, P., Walker, O., Kirigia, J., Mwikisa, C., \& Mbeeli, T. (2010). Inequities in utilization of maternal health interventions in Namibia: Implications for progress towards MDG 5 targets. International Journal for Equity in Health, 9(1), 1-11.

Publisher's Note Springer Nature remains neutral with regard to jurisdictional claims in published maps and institutional affiliations. 